\title{
Hydration Effects in Exchange Reactions and Mineral Growth: Lessons from Glassy Systems
}

\author{
RICHARD C. REMSING ${ }^{1}$ \\ ${ }^{1}$ Department of Chemistry and Chemical Biology, Rutgers \\ University, Piscataway, NJ 08854
}

In recent years, it has become apparent that water and dissolved ions can be key players in driving fundamental geochemical processes. I will discuss the non-trivial role of the solvent in exchange reactions, a fundamental geochemical process at the molecular scale relevant to mineral growth and dissolution. I will describe how we can borrow and extend concepts from condensed matter physics and combine them with computer simulations to make predictions that connect with experiment data. In particular, I will focus on the complex dynamics of exchange reactions involving ionic species. These exchange reactions are a key step of solutionphase mineral growth and dissolution, such that the kinetics of exchange reactions strongly correlate with mineral growth and dissolution rates. However, the extreme sensitivity of exchange rates to changes in the nature of an ion is not well understood. To explain this behavior, we borrow concepts from the physics of glassy systems and demonstrate how the experimentally-observed scaling of exchange rates with ionwater interaction strength can be predicted from knowledge of intermolecular interactions. Extensions of these concepts to multi-scale models of mineral growth will also be discussed. These findings provide new insights into basic molecularscale geochemical processes and illustrate how progess can be made by connecting with other disciplines. 